\title{
Treatment of Oral Leukoplakia with Diode Laser: a Pilot Study on Indian Subjects
}

\author{
Usama A Rashid Kharadi' ${ }^{1 *}$, Sanjeev Onkar², Rajendra Birangane ${ }^{3}$, Swapnali \\ Chaudhari $^{2}$, Abhay Kulkarni ${ }^{4}$, Rohan Chaudhari ${ }^{4}$
}

\begin{abstract}
Background: To evaluate the safety, convenience and effectiveness of 940nm diode laser for treatment of homogenous leukoplakia. Materials and Methods: Ten patients having homogenous leukoplakia which were diagnosed clinically were selected from an Indian dental educational institution for the study. Toludine blue staining was applied locally over the lesion. The area where there was increased uptake of stain was excised using a 940 nm EZLASE TM diode laser (BIOLASE-USA). Results: Although various treatment modalities have been tried and the search continues for novel treatment modalities for complete removal of homogenous leukoplakia, from results of our preliminary pilot study it is clear that the use of $940 \mathrm{~nm}$ diode laser as a treatment modality for homogenous leukoplakia is a good substitute. Healing was perfect without any complication within a duration of 1 month. Pain intensity was also mild and absolutely zero on the VAS scale after 1 month follow up. Conclusions: $940 \mathrm{~nm}$ diode lasers are safe and can be effectively used as a treatment modality of homogenous leukoplakia, without any complication and without compromising health and oral function of patients. Considering recurrence factor, long term follow up for patients is a must.
\end{abstract}

Keywords: $940 \mathrm{~nm}$ diode laser - homogenous leukoplakia - surgical treatment

Asian Pac J Cancer Prev, 16 (18), 8383-8386

\section{Introduction}

Cancer is said to be second most leading cause of mortality in economically developed countries following heart diseases, while in developing countries it is the third most leading cause of death following heart diseases and diarrheal diseases (Garcia et al., 2007). Oral cancer is the sixth most common cancer worldwide. According to the literature, Indian subcontinent shows the highest prevalence of oral cancer among all cancers in men (Brad et al., 2002). The researchers had well established the fact that almost all oral cancer are leaded by noticeable clinical changes in the form of white or red patches in oral mucosa \& set forth the theory of two-step process of cancer development (Reibel et al., 2003). These changes in oral cavity are labeled as "potentially malignant disorders (PMD) of oral cavity." The incidence and improving the survival of those who develop oral cancer depends on its Prevention, early detection \& successful treatment of preceding potentially malignant disorders (Fedele, 2009).

Leukoplakia is well accepted as potentially malignant disorders of oral cavity (Warnakulasuriya et al., 2007). Various studies showed the malignant transformation rate for leukoplakia ranges from 0.6 to $20 \%$ in different locations. Leukoplakia has been defined by various authors and many definitions are given to it. According to
Warnakulasuriya et al 2007 "Leukoplakia should be used to recognize white plagues of questionable risk having excluded (other) known diseases or disorders that carry no increased risk for cancer". The various locally acting etiological factors in development of leukoplakia are tobacco, alcohol, candidiasis, electrogalvanic reactions, ultraviolet radiation, herpes simplex and papilloma viruses. Leukoplakia is often related to tobacco addiction, while more than $80 \%$ of patients with leukoplakia are smokers. Smokeless tobacco usage is a deep-rooted fact for the development of leukoplakia. Clinically Oral leukoplakia is seen as white lesion of more than $5 \mathrm{~mm}$ which cannot be scraped off. It is more habitually found in men, it occurs on mucosal surface, and rarely causes uneasiness or pain. Approximately $70 \%$ of oral leukoplakia lesions are found on the buccal mucosa, vermilion border of the lower lip, and on gingiva. There is no consensus regarding the most appropriate treatment for oral leukoplakia. Presently it has multiple treatment modalities such as surgical and conservative methods. The commonly used conservative modalities are antifungal ointments, glycerin soap massage, vitamin A supplements, Antioxidants. The surgical modality should be considered if the lesion doesn't resolve, even after stoppage of addiction \&/or medical treatment. The most common surgical method is complete excision of lesion. Conventional surgeries 
even though routinely performed have few disadvantages. Diode laser is commonly used as a surgical soft tissue laser $\&$ for the same reason here we have done a short study to evaluate the success of diode laser in surgical treatment of homogenous leukoplakia.

\section{Objectives}

The present pilot study was designed to evaluate the safety and efficacy of $940 \mathrm{~nm}$ diode laser for treatment of homogenous leukoplakia. The objectives were to evaluate the healing of lesion and pain intensity at different time interval.

\section{Materials and Methods}

The study was conducted at Pandit Deendayal Upadhyay Dental College \& Hospital, Solapur, India, in the department of Oral Medicine \& Radiology. Patients having homogenous leukoplakia which were diagnosed clinically by experts in oral medicine were selected for

Table 1. Distribution of Subjects According to age and Gender

\begin{tabular}{llcc}
\hline Variables & & Frequency & Percentage \\
\hline Age in Years & $30-45$ & 6 & $60 \%$ \\
\multirow{3}{*}{ Gender } & $46-60$ & 4 & $40 \%$ \\
& Male & 8 & $80 \%$ \\
& Female & 2 & $20 \%$ \\
\hline
\end{tabular}

Table 2. Distribution of Homogenous Leukoplakia According to Anatomic Subsides

\begin{tabular}{llcccc}
\hline $\begin{array}{l}\text { Serial } \\
\text { number }\end{array}$ & Gender & $\begin{array}{c}\text { Labial } \\
\text { mucosa }\end{array}$ & $\begin{array}{c}\text { Anterior } \\
\text { buccal } \\
\text { Mucosa }\end{array}$ & $\begin{array}{c}\text { Posterior } \\
\text { buccal } \\
\text { Mucosa }\end{array}$ & Recurrence \\
\hline 1 & Male & - & - & $\mathrm{P}$ & - \\
2 & Male & - & $\mathrm{P}$ & - & - \\
3 & Female & - & - & $\mathrm{P}$ & $\mathrm{P}$ \\
4 & Male & - & $\mathrm{P}$ & - & - \\
5 & Male & - & - & $\mathrm{P}$ & - \\
6 & Female & P-Lower & - & - & - \\
7 & Male & - & - & $\mathrm{P}$ & $\mathrm{P}$ \\
8 & Male & P-Lower & - & - & - \\
9 & Male & - & - & $\mathrm{P}$ & - \\
10 & Male & - & - & $\mathrm{P}$ & - \\
\hline
\end{tabular}

Table 3. Healing of Lesion After Diode Laser Excision at Different Time Interval According with Landry et al Healing Index

\begin{tabular}{|c|c|c|c|c|}
\hline \multirow[t]{2}{*}{ Subjects } & \multicolumn{3}{|c|}{ Healing } & \multirow{2}{*}{$\begin{array}{l}\text { Recurrence after } \\
6 \text { month follow-up }\end{array}$} \\
\hline & 15 days & 1 month & 3 month & \\
\hline 1 & 3 & 5 & 5 & No \\
\hline 2 & 3 & 5 & 5 & No \\
\hline 3 & 3 & 5 & 5 & Yes \\
\hline 4 & 2 & 4 & 5 & No \\
\hline 5 & 3 & 5 & 5 & No \\
\hline 6 & 3 & 5 & 5 & No \\
\hline 7 & 3 & 5 & 5 & Yes \\
\hline 8 & 2 & 5 & 5 & No \\
\hline 9 & 3 & 5 & 5 & No \\
\hline 10 & 3 & 5 & 5 & No \\
\hline
\end{tabular}

the study. Patients counseling was done \& explained them in detail about possible malignant behavior in homogenous leukoplakia. The patients were asked to completely quit the habit \& were kept on antioxidants $\&$ antifungal medications for 1 month duration. Those patients which showed response to conservative medical management were excluded from study while those who did not show any response to treatment were opted for laser use \& removal of lesion in toto. Toludine blue stain was applied locally with standardized method over the lesion. The area where there was increased uptake of stain was excised using $940 \mathrm{~nm}$ EZLASE TM diode laser, (BIOLASE-USA). The complete Procedure was explained to the patient and subsequently local infiltration was given around the lesion. Complete aseptic conditions were maintained and both patient and operator were given protective eyeglasses to wear as a protective measure. Patients on long term medications and those suffering from any other chronic debilitating diseases were not included in the study. Follow up of 4 visits was done (15 days, 1 month, 3 months and 6 months in succession) for

Table 4. Pain Intensity Measured According to Visual Analogue Scale (VAS) at Different Time Interval

\begin{tabular}{lccccc}
\hline Patient number & 7 days & 15 days & 1 month & 3 month & 6 month \\
\hline 1 & 4 & 2 & 0 & 0 & 0 \\
2 & 3 & 2 & 0 & 0 & 0 \\
3 & 4 & 2 & 0 & 0 & 0 \\
4 & 3 & 1 & 0 & 0 & 0 \\
5 & 4 & 2 & 0 & 0 & 0 \\
6 & 5 & 3 & 0 & 0 & 0 \\
7 & 3 & 1 & 0 & 0 & 0 \\
8 & 4 & 2 & 0 & 0 & 0 \\
9 & 4 & 2 & 0 & 0 & 0 \\
10 & 4 & 3 & 0 & 0 & 0 \\
\hline
\end{tabular}
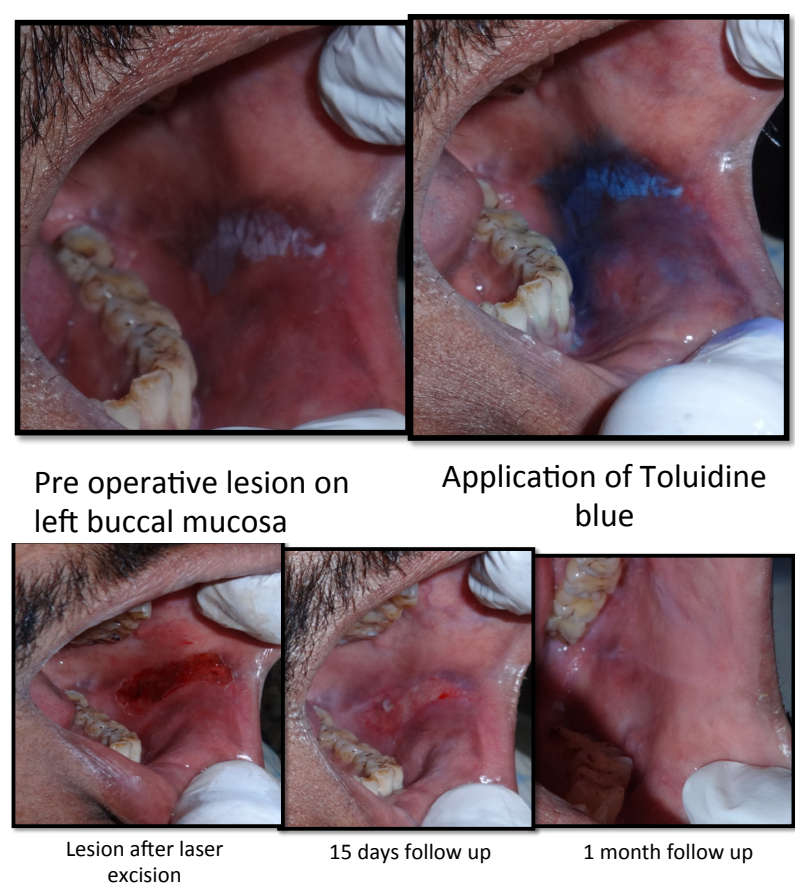

Figure 1. Patient with homogenous leukoplakia. Surgicall excision with Diode laser and follow up pictures 


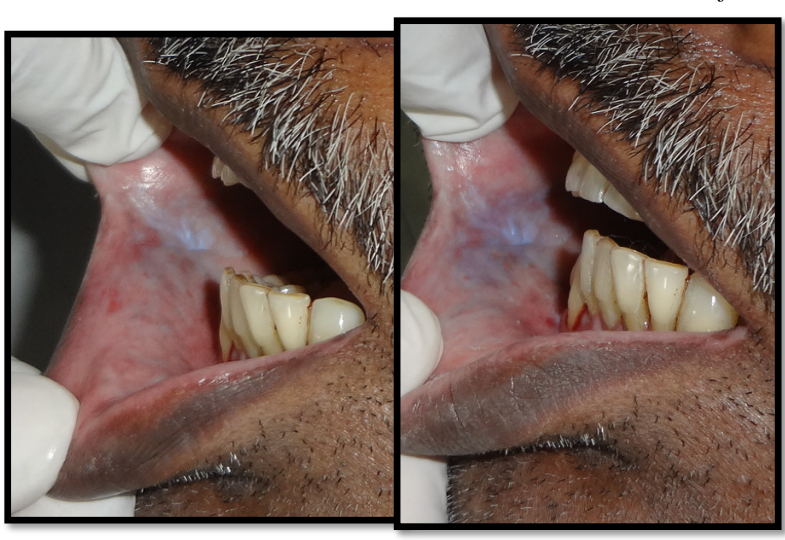

Pre operative lesion

on right buccal

mucosa

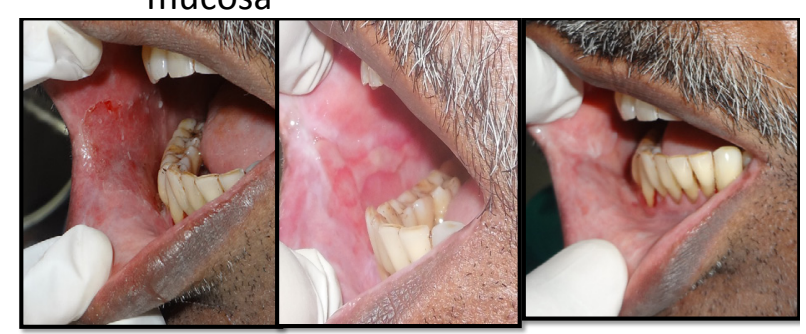

Lesion after laser 15 days follow up 1 month follow up excision

Figure 2. Patient clinically diagnosed as homogenous leukoplakia. Surical excision of lesion with Diode laser and follow up pictures

post operative pain, healing, recurrence and malignant transformation if any. Healing of lesion was measured according to healing index given by Landry et al and pain intensity measured on Visual analogue scale (VAS). The institutional ethical committee approved study protocol. A detailed history from the subjects was obtained regarding their general health, oral hygiene and previous adverse habits, nature of etiologic agent, duration of use, site of placement and any other associated deleterious habits. An informed consent was obtained from all the participants.

\section{Results}

Out of 10 patients with homogenous leukoplakia treated with diode laser, 8 patients showed complete disappearance of lesion even after 6 months follow up. Two patients showed recurrence of lesion on same location in mild form which was persistent but not growing in size.

\section{Discussion}

Light amplification by stimulated emission of radiation (LASER) had proved itself in many areas of medicine \& dentistry. This perticular technology has developed very speedily \& proper understanding of laser interaction with humans, has expanded the scope for its uses (Sahar-Helft, 2011). The various advantages of lasers over conventional surgical procedures using scalpel, such as painless procedure, bloodless field, less post operative pain, comfortable for patients had made it more popular and are now a days used routinely by general medical and dental practitioners. Laser have been tried \& successfully used in many areas of dentistry such as for incisional \& excisional biopsy, for surgical removal of tongue lesions, red \& white lesions, treating potentially malignat disorders (PMD) of oral cavity, vesiculobullous lesions, salivary gland pathologies, apthous \& herpetic ulcers, treatment and prevention of radiation mucositis, coagulation of bleeding areas, periodontal surgeries, prosthetic \& endodontic applications (Rohit Malik et al., 2011). The conservative management is often advised after diagnosing PMD of oral cavity. The results are often good. Those lesions that are reluctant to medical therapy, has to go for surgical excision to avoid further damage. Conventional surgeries using scalpel has its own advantages \& disadvantages. Recently lasers have also been tried as a treatment modality for PMD \& amazing results were proved. Active medium in diode lasers is a solid semiconductor with variable wavelengths between 800 and $980 \mathrm{~nm}$. This particular wavelength is inadequately absorbed by the dental hard tissues, that's why diode laser is safe and well prescribed for oral soft tissue surgeries rather than hard tissues (Nihat et al., 2013). The fact that incidence of PMD is somewhat more in rural areas where there is often lack of advanced machinery and clinicians are not very experienced in using such machines. Most of the patients visiting in our institute are from rural and semi urban areas. Though initial cost of laser machine is high, at an institutional level laser is cost effective. Hence we agreed for the use of $940 \mathrm{~nm}$ diode laser for our patients due to its availability, ease of its function, bloodless field, the ability to treat large areas to in a single application, and the possibility of exact control of laser fluency in all areas of the mouth.

According to literature, recurrence and malignant transformation from the lesion have occasionally been observed following laser surgery (Ishii et al., 2003). In our pilot study patients were followed for up to 6 months and none of the patients showed malignant transformation. However, in 2 patients (one male and one female, both during 3-6 months follow up) recurrence in mild form was observed. According to Marek VIK (2013), women's are at high risk in malignant conversion of leukoplakia. Out of 10 patients selected in our study, the mean age group of patients was 44.2 years and $80 \%$ patients were male while $20 \%$ were female (Table 1 ). This can be a possibility that none of our patients turned into malignancies as females sample size was less. Patients were followed for checking wound healing post operatively according to Landry et al index (Table 3) and for measuring of pain intensity as per visual analogue scale (VAS) (Table 4). Mild post operative pain was there but it doesn't cause any obstacle in routine function of patient which was completely zero on 1 month follow up. The adjacent oral soft tissues showed no complications. Literature on lasers are vast and recent studies like Tatu et al. (2013) treated patient having homogenous leukoplakia with diode laser and concluded that diode laser as an alternative tool for surgical management of various potentially malignant lesions of oral cavity (Tatu et al., 2013). Nihat et al. (2013) treated 27 patients who had different benign oral lesions with the $810 \mathrm{~nm}$ diode laser and concluded that diode laser may indeed be the best choice in oral soft tissue surgery. 


\section{Usama A Rashid Kharadi et al}

Goharkhay et al. (1999) in their study concluded that the diode laser is a very effective and because of its excellent coagulation ability, it is a useful alternative in soft-tissue surgery of the oral cavity (Goharkhay et al., 1999). Marek Vlk, Roman Smucle (2013) concluded that malignant transformation of leukoplakia does occur even after laser ablation, and for this reason it is necessary to subject the patients to systematic follow-ups. It is significantly higher risk of recurrences and malignant transformation in tongue and oral base. Laser ablation is a simple and well-tolerated procedure with high clearing rate (Marek et al., 2013). Kruti A Shah (2014) concluded that diode laser provides good coagulation, healing, reduces surgical time and prevents high grade infection (Kruti et al., 2014).

There are many controverseries regarding malignant transformation of leukoplakia lesion after surgical laser excision. Various studies failed to showed malignant transformation after laser therapy while other studies concluded that laser ablation of lesion prevents malignant transformation. Definitely there is correlation between location of lesion, dysplastic activity, adverse habits, gender, and presence of lesion for longer duration, immunity of patient. In our study we had divided total 10 patients according to anatomic location of lesion into 3 groups (Table 2). $30 \%$ of lesion was at labial mucosa which showed complete disappearance of lesion on follow up period without any recurrence even after 6 months follow up. $28.57 \%$ of lesion was on anterior buccal mucosa which also does not showed recurrence, while remaining $71.42 \%$ lesions on posterior buccal mucosa which showed recurrence. Thus we can say that definitely there is association between location of lesion and its recurrence after surgery.

In Conclusion, within the limits of our present preliminary study, it is possible to conclude that $940 \mathrm{~nm}$ diode laser can safely \& effectively used as a treatment modality for homogenous leukoplakia, without any complication \& without compromising health and function of patients. Patients should be followed for extensive time period to confirm the recurrence or malignant transformation of homogenous leukoplakia. Large-scale longitudinal studies needed for further evaluation of the results.

\section{References}

Brad W, Neville (2002). Oral cancer and precancerous lesions. CA Cancer J Clin, 52, 195-215.

Fedele $\mathrm{S}$ (2009). Diagnostic aids in the screening of oral cancer. Head Neck Oncol, 1, 5-11.

Garcia M, Jemal A, Ward EM, et al (2007). Global cancer facts and figures 2007. American Cancer Society.

Goharkhay K, Moritz A, Wilder-Smith P, et al (1999). Effects on Oral Soft Tissue Produced by a Diode Laser In Vitro. Lasers Surg Med, 25, 401-6.

Ishii J, Fujita K, Komori T (2003). Laser surgery as a treatment for oral leukoplakia. Oral Oncol, 39, 759-69.

Kruti A Shah, Hemal R Brahmkshatriya, Rushit J Patel, Mansi H Brahmkshatriya (2014). Clinical and histopathological evaluation of healing after excision of leukoplakia with diode laser-research article. Adv Hum Biol, 4, 45-49.

Marek VIK, Roman Smucle (2013). The efficacy of diode laser removal of leukoplakias on the tongue and in lower buccal cavity compared to other buccal cavity locations-A two-year study. Central Eur J Med, 8, 192-203.

Nihat Akbulut, Sebnem Kursun E, Ugur Gulsen (2013). Is the 810 -nm diode laser the best choice in oral soft tissue therapy? Eur J Dentistry, 7, 207-211.

Reibel J (2003). Prognosis of oral pre-malignant lesions: significance of clinical, histopathological, and molecular biological characteristics. Crit Rev Oral Biol Med, 14, 47-62.

Rohit Malik, LK Chatra (2011). Laser an inevitable tool in modern dentistry: An overview. J Indian Acad Oral Med Radiol, 23, 603-8.

Sahar-Helft S (2009). The laser technology in dentistry. Refuat Hapeh Vehashinayim, 26, 12.

Tatu R, Shah K, Palan S, et al (2013). Laser excision of labial leukoplakia with diode laser: A case report. Indian J Res Reports Med Sci, 3, 64-66.

Warnakulasuriya S, Newell W, Van der Waal I, et al (2007). Nomenclature and classification of potentially malignant disorders of the oral mucosa.J Oral Pathol Med, 36, 575-80. 